Int. J. Electrochem. Sci., 14 (2019) $2732-2742$

\title{
Direct Electrochemistry and Electrocatalysis of Myoglobin with Copper Benzenetricarboxylate Metal-Organic Frameworks@ Nitrogen-Doped Graphene Composite Modified Electrode
}

\author{
Guiling Luo ${ }^{1}$,Ying Deng ${ }^{1}$,Hui Xie ${ }^{1}$, Juan Liü ${ }^{2}$, Si Mi ${ }^{1}$, Binghang $L^{1}$, Guangjiu Li ${ }^{2}$, Wei Sun ${ }^{1, *}$ \\ ${ }^{1}$ Key Laboratory of Functional Materials and Photoelectrochemistry of Haikou, Key Laboratory of \\ Laser Technology and Optoelectronic Functional Materials of Hainan Province, College of Chemistry \\ and Chemical Engineering, Hainan Normal University, Haikou 571158, P R China \\ ${ }^{2}$ Key Laboratory of Optic-electric Sensing and Analytical Chemistry for Life Science of Ministry of \\ Education, College of Chemistry and Molecular Engineering, Qingdao University of Science and \\ Technology, Qingdao 266042, P R China \\ *E-mail: sunwei@hainnu.edu.cn
}

doi: $10.20964 / 2019.03 .39$

Received: 19 November 2018 / Accepted: 11 January 2019 / Published: 7 February 2019

\begin{abstract}
A novel nanohybrid material based on copper benzenetricarboxylate metal-organic framework $(\mathrm{Cu}-$ BTC) and nitrogen-doped graphene (NG) was used to immobilize myoglobin (Mb) with its biosensing ability checked. The surface images of the modifiers on the electrode were characterized by SEM. Spectroscopic results demonstrated that $\mathrm{Mb}$ kept its native structure in the composite film. Direct electrochemistry of $\mathrm{Mb}$ on Cu-BTC@NG modified electrode was checked by cyclic voltammetry with a pair of well-defined redox peaks observed. The peak-to-peak separation of $0.082 \mathrm{~V}$ and the formal peak potential of $-0.187 \mathrm{~V}$ were calculated, corresponding to the realization of direct electrode transfer of $\mathrm{Mb}$. Electrochemical investigations of Mb modified electrode were studied in detail. The modified electrode showed good sensitivity and wide linear range to the analysis of trichloroacetic acid and sodium nitrite. All the results demonstrated that is hybrid matrix exhibited a fast electron transfer pathway for the electron transfer of $\mathrm{Mb}$.
\end{abstract}

Keywords: Copper benzenetricarboxylate metal-organic framework; nitrogen-doped graphene; myoglobin; direct electrochemistry

\section{FULL TEXT}

(C) 2019 The Authors. Published by ESG (www.electrochemsci.org). This article is an open access article distributed under the terms and conditions of the Creative Commons Attribution license (http://creativecommons.org/licenses/by/4.0/). 